\title{
Postoperative atrial fibrillation: Adding (fish) oil to the fire
}

\author{
Niv Ad, MD, ${ }^{a, b}$ and Paul S. Massimiano, MD
}

\footnotetext{
From the ${ }^{\mathrm{a} D e p a r t m e n t}$ of Cardiovascular and Thoracic Surgery, West Virginia University, Morgantown, WVa, and ${ }^{\mathrm{b}}$ Adventist HealthCare, Takoma Park, Md.

Disclosures: Dr Ad is a consultant for Medtronic, a member of the speaker's bureau for AtriCure, proctor and member of the speaker's bureau for LivaNova, on the advisory board for Nido Surgical, and co-owner for Left Atrial Appendage Occlusion, LLC. Dr Massimiano has nothing to disclose with regard to commercial support.

Received for publication Sept 14, 2017; accepted for publication Sept 15, 2017; available ahead of print Oct 25, 2017.

Address for reprints: Niv Ad, MD, Department of Cardiovascular and Thoracic Surgery, West Virginia University, 1 Medical Center Dr, Morgantown, WV 26506 (E-mail: nivadmd14@gmail.com).

J Thorac Cardiovasc Surg 2018;155:252-3

$0022-5223 / \$ 36.00$

Copyright (c) 2017 by The American Association for Thoracic Surgery

https://doi.org/10.1016/j.jtcvs.2017.09.078
}

The study by Akintoye and colleagues ${ }^{1}$ published in this issue of the Journal, "Factors Associated with Postoperative Atrial Fibrillation and Other Adverse Events After Cardiac Surgery," is a second look at the results from the Omega-3 Fatty Acids for Prevention of Post-operative Atrial Fibrillation (OPERA) trial, a randomized, doubleblind, placebo-controlled, multinational, clinical trial that tested the potential impact of fish oil on the incidence of postoperative atrial fibrillation. ${ }^{2}$ The study was an international one with centers from 3 countries (United States, Argentina, and Italy) and included 1516 patients enrolled in 28 centers. The findings of the OPERA trial showed that perioperative supplementation with n-3-polyunsaturated fatty acids did not reduce the risk of postoperative atrial fibrillation.

This new analysis published in this issue of the Journal was set to evaluate the incidence and the potential impact of postoperative atrial fibrillation and other perioperative morbidities on major adverse cardiac events (MACE). Randomized controlled trials are not so common in cardiac surgery, and the leaders behind this study should be congratulated. In the original design of the study, there are a few points that are worth recognizing, as they may have impacted the overall results. First, the study design allowed for patients with a history of atrial fibrillation and antiarrhythmic therapy to be included. Although the treatment groups were somewhat balanced, when it comes to history of atrial fibrillation and other supraventricular arrhythmias, this decision may be considered somewhat controversial, because the endpoint of the study was the incidence of postoperative atrial fibrillation and the potential impact of fish oil. Moreover, it is unclear how the history of atrial fibrillation was captured, and the potential differences between practices in different centers and countries may have had some unknown impact.

Second, the definition of atrial fibrillation for the primary endpoint was any event longer than 30 seconds, which is taken from the Heart Rhythm Society guidelines ${ }^{3}$ and are as follows: mortality. MACE.

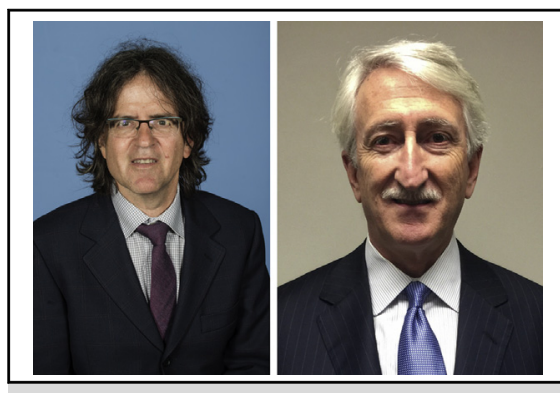

Niv Ad, MD, and Paul S. Massimiano, MD

Central Message

The findings shed light on the incidence of atrial fibrillation in a randomized trial. Future studies should further define the nature of postoperative atrial fibrillation and its impact under varied clinical scenarios.

See Article page 242

probably has nothing to do with the clinical aspects of postoperative atrial fibrillation. This decision may have influenced the outcome of the study, although the number of recurrent events and occurrences of atrial arrhythmia that required treatment were captured as secondary endpoints and found not to be impacted by the treatment as well. Setting the threshold for atrial fibrillation for 30 seconds probably impacted the number of events captured and may have had some implications when the final analysis of the study was done.

The main findings of this subanalysis of the OPERA trial

1. Postoperative atrial fibrillation was associated with the type of surgery and the use of cardiopulmonary bypass.

2. Concomitant valve procedures and coronary artery bypass grafting (CABG) carry high risk for MACE and

3. Compared with spontaneous return of rhythm, assisted ventricular pacing was associated with greater rates of

4. Postoperative atrial fibrillation resulted in increased resource use and 1-year mortality.

Throughout the years, various reports and clinical practice associated the type of surgery with the incidence of postoperative atrial fibrillation. This study is unique because it is a prospective, randomized trial in which the findings are based on multiple centers from different countries. This in fact reconfirms that the risk for postoperative 
atrial fibrillation shares global variables such as the type of procedure. The reports from the Society of Thoracic Surgeons (STS) database do show that the type of surgery is associated with the risk to develop postoperative atrial fibrillation with CABG and valve surgery. In the reports from 2014 to 2016, the rates of new-onset postoperative atrial fibrillation for CABG and mitral valve replacement were the greatest $(43.8 \%, 44.2 \%$, and $45.9 \%$, respectively). Similarly, the incidence for CABG was lower compared with valve and concomitant valve and CABG surgeries. Careful reading of the study reveals that age and sex were highly associated with these findings. Several important studies associated clinical variables such as age, sex, chronic obstructive pulmonary disease, vascular disease, and renal failure with postoperative atrial fibrillation; therefore, focusing on the type of procedure only may lead to a significant bias. ${ }^{4}$

The association between the rate of MACE, the type of procedure, and ventricular pacing likely warrants a more careful look. The study was done in different institutions and countries with multiple varying periprocedural protocols that even the best randomization scheme is unable to control. The use of validated tools to predict procedural outcome has the potential to significantly improve our understanding of such events. The STS risk score and the EuroSCORE II are widely used and relatively reliable in such predictions. In general, patients having a combined $\mathrm{CABG}$ and valve procedure are older and sicker; therefore, greater rates of complications are expected. The importance of the study discussed is its international affiliations; this is why the use of such scores such as STS and EuroSCORE may shed more light on the practice, patient selection, and performance across the study centers.

Postoperative atrial fibrillation is an important complication after cardiac surgery, and it is not surprising that it was found to be associated with greater resource use and 1-year mortality. The challenge we are all having is how to delineate and isolate the impact of atrial fibrillation postoperatively. As the study pointed out, the type of procedure greatly influenced the incidence of atrial fibrillation. In contrast, patients for concomitant valve and CABG are the oldest and sickest of our patients with the greatest resource use and 1-year mortality, so maybe postoperative atrial fibrillation should be considered as a marker for a sick patient rather than the cause of the complications and the greater cost. More so, despite years of research, we have yet to have a study looking into the specifics of postoperative atrial fibrillation in terms of the different clinical scenarios. Maybe we should try to isolate the event of postoperative atrial fibrillation to better understand the potential impact. Let's look into 2 different clinical situations:

1. A patient who is in the intensive care unit, intubated with low cardiac output on multiple inotropes, who develops atrial fibrillation on day 2 postoperatively.

2. A patient who is on the floor after an uneventful surgery, ambulating well, who develops atrial fibrillation on day 2 postoperatively.

It is clear that in a study or a registry these 2 patients will be captured as having postoperative atrial fibrillation, but it is obvious that the clinical situation and potential impact of the complication are significantly different and should not be approached as only statistics. We have to develop a better understanding and be significantly more granular in approaching postoperative atrial fibrillation when it comes to primary prevention, management, and diminishing the risk for complications.

In summary, the findings of this international study shed some light on the incidence of atrial fibrillation in a large, multicenter, prospective randomized trial. The study is important and improves the evidence in the field. Future studies should focus on further defining the nature of postoperative atrial fibrillation and its true impact under different clinical circumstances.

\section{References}

1. Akintoye E, Sellke F, Marchioli R, Tavazzi L, Mozaffarian D. Factors asso ciated with postoperative atrial fibrillation and other adverse events after cardiac surgery. J Thorac Cardiovasc Surg. 2018;155:242-51.e10.

2. Mozaffarian D, Marchioli R, Macchia A, Silletta MG, Ferrazzi P, Gardner TJ, et al; OPERA Investigators. Fish oil and postoperative atrial fibrillation: the Omega-3 Fatty Acids for Prevention of Post-operative Atrial Fibrillation (OPERA) randomized trial. JAMA. 2012;308:2001-11.

3. Calkins H, Kuck KH, Cappato R, Brugada J, Camm AJ, Chen SA, et al; Heart Rhythm Society Task Force on Catheter and Surgical Ablation of Atrial Fibrillation. 2012 HRS/EHRA/ECAS expert consensus statement on catheter and surgical ablation of atrial fibrillation: recommendations for patient selection, procedural techniques, patient management and follow-up, definitions, endpoints, and research trial design. Heart Rhythm. 2012;9:632-96.

4. Creswell LL, Schuessler RB, Rosenbloom M, Cox JL. Hazards of postoperative atrial arrhythmias. Ann Thorac Surg. 1993;56:539-49. 